Citation: Diana S. (2021) Rewriting Marx to expose the data society and $A l$, in «Cambio. Rivista sulle trasformazioni sociali», Vol. 11, n. 21: 199-211. doi: 10.36253/cambio-10637

Copyright: (c) 2021 Diana S. This is an open access, peer-reviewed article published by Firenze University Press (http://www.fupress.com/cambio) and distributed under the terms of the Creative Commons Attribution License, which permits unrestricted use, distribution, and reproduction in any medium, provided the original author and source are credited.

Data Availability Statement: All relevant data are within the paper and its Supporting Information files.

Competing Interests: The Author(s) declare(s) no conflict of interest.

\section{Rewriting Marx to expose the data society and AI}

\author{
Stefano Diana \\ independent researcher \\ stefanodiana.inc@gmail.com
}

\begin{abstract}
As I show with examples from the Grundrisse and the Capital, some fundamentals of Marx's critique of political economy can be rewritten with simple substitutions to yield surprisingly pertinent analyses of today's "information society" and the role that data and AI play in it. The reason behind this strange phenomenon is that Marx's penetrating study of money as a dehumanizing abstraction and self-replicating capital can be extended with minor changes to the main abstraction and "automatic fetish" of our historical regime: digital data. As money-capital tends to grow by itself independently of humans, so does data. AI is the main engine of this new dangerous cycle.
\end{abstract}

Keywords: digital data, artificial intelligence, information society, automatic fetish, personal knowledge.

I can behave humanely with the thing, only if the thing behaves humanely with me. Karl Marx

Besides, I hate everything that only instructs me, without immediately increasing or enlivening my activity.

Johann Wolfgang von Goethe

\section{FOREWORD}

This work has nothing to do with Marxism. It's not a new application of Marx's theories. It does not belong to the strand of studies on contemporary adaptations of Marx's theories to our age, e.g. revised definitions of "general intellect" or compositions of capital. It is more of using Marx's style of thought to read the culture we live in.

I'm only referring here to a small slice of Marx (\&Engel)'s analysis, dealing with the social meaning and effects of money becoming capital. I will use it by analogy to explore the social meaning and effects of digital 
data, and of those special data computing techniques known as Artificial Intelligence (AI). However, since that slice is an integral part of Marx's socio-economic critique, the restrained instance presented in this paper can be considered a proof of concept and a starting point of a much broader investigation that might be undertaken.

\section{A FIRST REWRITE}

I propose an experiment, which at first glance might seem an exercise in Raymond Queneau's "potential literature". Let's start from this piece of Marx's Grundrisse as an example.

Every particular commodity, in so far as it is exchange value, has a price, expresses a certain quantity of money in a merely imperfect form, since it has to be thrown into circulation in order to be realized, and since it remains a matter of chance, due to its particularity, whether or not it is realized. However, in so far as it is realized not as price, but in its natural property, it is a moment of wealth by way of its relation to a particular need which it satisfies; and, in this relation, [it] expresses (1) only the wealth of uses, (2) only a quite particular facet of this wealth. Money, by contrast, apart from its particular usefulness as a valuable commodity, is (1) the realized price; (2) what satisfies every need, in so far as it can be exchanged for the desired object of every need, regardless of any particularity. The commodity possesses this property only through the mediation of money. Money possesses it directly in relation to all commodities, hence in relation to the whole world of wealth, to wealth as such. With money, general wealth is not only a form, but at the same time the content itself. The concept of wealth, so to speak, is realized, individualized in a particular object. In the particular commodity, in so far as it is a price, wealth is posited only as an ideal form, not yet realized; and in so far as it has a particular use value, it represents merely a quite singular facet of wealth. In money, by contrast, the price is realized; and its substance is wealth itself considered in its totality in abstraction from its particular modes of existence (Marx 1857-58 [1973]).

What is Marx saying here? Commodities exist as tangible things because they are good for something and for someone. They might be exchanged, and therefore have an exchange value. But essentially a commodity «in its natural property» has only a particular and concrete "use value" for the one who needs and uses it. This concrete and personal character means that commodities originally have no connection with the abstract concept of "wealth". The idea of wealth is meaningless when commodities are made by a man for his own needs, that is where «the extent of his production is measured by his need» (Marx 1844).

Money instead transcends any personal and peculiar use value of any commodity: it is an abstract representation, like a universal unit of measurement for the "exchange value" of different commodities between people. In fact, we can consider the market as a measuring device that converts everything to numbers. While generating those "exchange values", Marx notes, money gradually gains its independence and turns into the universal mediator between men and goods, that is between men and their own desires, and eventually between man and man. ${ }^{1}$ At the same time, the ideal concept of "wealth" becomes real: it's an amount of money.

Around the middle of last century, the scientist and philosopher Michael Polanyi carried out one of the first modern and realistic inquiries into how we create and shape knowledge (Polanyi 1958[1966]). I say "modern and realistic" because it builds on the actual capabilities of our bodies—on the «bodily roots of all thought», he saysand on up-to-date science, instead of adding yet another old-school speculation on words and abstract concepts, far from scientific grounds. Marx is modern and realistic likewise: he always looked at history and concrete life conditions of real people with real bodies and sentiments, in order to develop his social theory. It's no coincidence that mathematics and formulas are rare in Marx's political economy, what matters to him is the real story told. In present academic economics, by contrast, papers without math are unacceptable.

Polanyi's description of knowledge, which is aligned to the subsequent findings of cognitive psychology and neuroscience, is at the heart of my Marx rewrite. Polanyi noticed how «we know more than we can express». This "tacit/personal knowledge" is the primary knowledge embodied in a particular subject, accumulated over years of her unique life experience, always practical as much as theoretical. It's a procedural knowledge inscribed in dif-

\footnotetext{
${ }^{1}$ «Money is the procurer between man's need and the object, between his life and his means of life. But that which mediates my life for me, also mediates the existence of other people for me» (Marx 1844).
} 
ferent systems of our organisms. It's the uncountable deeds we can do but can't explain how, such as recognizing a familiar face, with all its meaning, or catching a frisbee on the fly. It's the experts' know-how: physicians, film directors, athletes, artisans, chefs, scientists, artists, etc. It's the place where the spark of novel ideas and solutions comes from. It's the «secret region from which what must be thought gushes», as the poet Heidegger would say. In short, it's the very foundation of all knowledge, which is unconscious and ineffable.

Let's now try and rewrite Marx's quote, taking care to replace

- "commodity" with (lived, subjective, tacit) "personal knowledge";

- "price" with "information";

- "use" with "experience";

- "money" with (digital) "data";

- "wealth" with "knowledge" in a general, abstract, impersonal sense.

Here is the result:

* Every particular personal knowledge, in so far as it can be exchanged, is information, expresses a certain quantity of data in a merely imperfect form, since it has to be thrown into circulation in order to be realized, and since it remains a matter of chance, due to its particularity, whether or not it is realized. However, in so far as it is realized not as information, but in its natural property, it is a moment of general knowledge by way of its relation to a particular need which it satisfies; and, in this relation, [it] expresses (1) only the knowledge of experience, (2) only a quite particular facet of this general knowledge. Data, by contrast, apart from its particular usefulness as a valuable personal knowledge, is (1) the realized information; (2) what satisfies every need, in so far as it can be exchanged for the desired object of every need, regardless of any particularity. Personal knowledge possesses this property only through the mediation of data. Data possesses it directly in relation to all instances of personal knowledge, hence in relation to the whole world of knowledge, to general knowledge as such. With data, abstract knowledge is not only a form, but at the same time the content itself. The concept of knowledge, so to speak, is realized, individualized in a particular object. In the particular personal knowledge, in so far as it is information, knowledge is posited only as an ideal form, not yet realized; and in so far as it has a particular experiential value, it represents merely a quite singular facet of general knowledge. In data, by contrast, information is realized; and its substance is knowledge itself considered in its totality in abstraction from its particular, personal modes of existence.

Let's check out the meaning and relevance of this rewrite.

- Following Polanyi, human knowledge is originally embodied in a particular person. For this person, knowledge has an existential value: it is an integral part of what life means to her. Subjective knowledge is isolated from shared, social knowledge: it has to find an expression and become explicit knowledge in order to "realize" itself in some way. It may be then exchanged between bodies without language: for example, showing how to crack a nut. Or it may be put into some language, with different degrees of ambiguity.

- As commodities satisfy "needs" through "use", so do experiences that become personal knowledge, since we need it to live.

- As "price" is the converted form of a commodity into a number, so "information" is the converted form of some knowledge into a number. Being sheer numbers, both representations have the maximum level of abstraction. Like price to commodities, information has only a vague relationship with personal experiences and knowledge, in a "merely imperfect form", and it's not an inherent property of theirs.

- Digital data is information, and it is "realized knowledge" insofar as it is the definitive form which every kind of knowledge tends to, in the digital culture, like everything tends to be represented by money in the capitalist market culture.

- Data itself can be an object of experience, and thus "valuable personal knowledge". In that role, data is commonly called "digital content". In fact, data is not only a form for content, but also content itself at the same time, as it can be experienced, exchanged, traded, processed, transformed into other data, and the latter data can be consumed as content or processed again, in an unlimited chain.

- Just as almost all can be translated into money, so almost all can be translated into data, by means of human action or sensors. But data abstracts from any particular aspect of the world, from any type of information, from any personal knowledge. All things datified are the same: a movie, a symphony, a diary, a medical record, 
a protein model, a shopping list, in the end they are all bit sequences. In this sense, today many believe that data, taken as a whole, can represent "knowledge realized". The body of data contained in the internet is increasingly regarded as the total of human knowledge, as if there were no relevant knowledge besides that.

- The datified knowledge is considered infinitely usable and fungible, precisely because it is represented in the universal code of digital data. The digital form is indifferent to content in the same sense a mathematical variable is; in fact, everything datified is the value of a numerical variable, for any chunk of bits is a number.

- Shared knowledge is explicit and tangible. When it becomes "information" and data, it's not only explicit and tangible, but also formalized. As such, it is both abstract from this or that particular subject, and also general, absolute. Humans tend to see any formalized stuff as objective and denatured knowledge, absolved of the original sins of subjectivity and ambiguity proper of human experience.

- As with the ideal concept of wealth, the ideal concept of knowledge is "realized" by digital data in the sense that it is made to coincide with a "particular object": the sequence of bits. A sequence of bit can be enjoyable content, metadata about that content, a program that can operate on other content or its metadata, metadata about a program, and so on.

\section{THE PHYLUM OF FORMAL THOUGHT}

We are now moving towards a condition in which knowledge is reduced to information, and information coincides with what is representable in digital data-that is, numbers. It can be seen as the final stage of a complex process that has shaped Western culture over a very long time, a process which is still active and which I call the big phylum of formalism. Here I will just review it briefly in three phases.

In the beginning there is Greek metaphysics, with its philosophical descendants. This school of thought spread and gave a shine to a specific method of inquiry and construction of knowledge which went mainstream in the next millennia. Here's how it works: arbitrarily isolate selected expressions from the flux and ambiguity of natural language; freeze them into closed and precise definitions; make them a transcendent matter; at the same time, hypostatize them so that they become in turn objects of investigation as if they were actual things of the physical world.

Every philosopher coined his/her trademark-words, starting with the universal metaphysical principles. "Being" is one of the first and most notable examples, as well as "Good", "Evil", "Truth", "Beauty”, "Freedom”, "Justice", and so on down to Heidegger's "Lichtung", etc. Turning a concept into an object through language triggers a telescopic stairway of abstractions: definitions built upon definitions built upon definitions... possibly endlessly. After some time, the arbitrary founding act is forgotten and the latest floors appear as well-founded knowledge. A bubble expands around that first nucleus, multiplying an incalculable error margin to its sustainable extreme. Until the inevitable collapse into reality.

This top-down method is shaped by the ignorance inherent in the human condition. Having a very limited insight into nature-including their internal nature-men have always reasoned about words, i.e. the only material they could freely dispose of, hoping to find in words the nature of things words should bespeak. However, language is a recent ability of the human body. Words are a quite late derivation of our sensitive experience and live on the fringes of our largely unconscious brain activity. Thus, the top-down method is desperately deceptive. It proceeds upside down (Changeux 2004) with respect to the natural history of evolution: as if words came first, and then things.

The phylum of formalism includes a wide variety of language games (Wittgenstein 1953),but they all share the practice of using common words as if they were mathematical entities.

We can see this shift as a murder of language (Barthes 1957): killing words to eliminate the ambiguity of their live usage. Classical logic, for example, builds on this act of killing: it makes sense only in a domain of dead words, terms with a fixed meaning. Despite its lack of plausibility as a model for human thought, logic has had an enormous cultural success thanks to the immediate advantages of its radical cognitive simplification. 
The second phase of formalism opens in Europe in $17^{\text {th }}$ century, driven by the explosion of mathematics as the primary cognitive medium. After an unprecedented blooming of math and science, the new idea gains ground that true knowledge is quantitative, general and abstract. On the other side, the old qualitative, concrete, material knowledge is downgraded to the remnants of a primitive, pre-scientific age, and falls into disgrace (Israel 1966).

The third and decisive leap of the formalist culture takes place in the $20^{\text {th }}$ century with the exceptional works of two mathematicians/engineers, Turing and Shannon, the former investigating the nature and limits of computability, the latter working to improve the transmission of messages on a physical channel. Both Turing's "machine" and Shannon's "message" are authentic mathematical entities, not metaphors. Turing's computation operates on abstract symbols with formal rules (Turing 1936-1937), while Shannon's information has a value that depends only on the probability distribution of its elements (Shannon 1948). Both computation and information are indifferent to their content (like variables, see above). They both do not consider meaning, the fundamental dimension of our existence and of communication between us human beings. ${ }^{2}$

Nevertheless, as with classical logic, the technical effectiveness of their results has caused Turing and Shannon's sphere of influence to expand uncontrollably in the territory of human affairs ${ }^{3}$. Turing's approach to computability came to assume an essential role in the definition of "intelligence" through the famous Turing test (Turing 1950), while Shannon's bit-encoded information tends now to represent all kinds of "information" and thus "knowledge".

The two aspects are related. The setting of the Turing test requires that the parties communicate only through texts. Beyond the original intentions, this experimental cunning has had considerable epistemological and anthropological bearings: it gave pseudo-scientific dignity to the belief that thought, consciousness, intelligence, are welldefined abstract abilities that can exist and be recognized without physical presence-that is, in the absence of a living body. And to the belief that information (digital data) is the very substance of that bodyless communication, and of those bodyless abilities.

In this way, the quite peculiar setting of the Turing test establishes a relationship between man and machine that is biased towards the machine right from the start. Two main outcomes derive from this.

1) The whole subsequent debate on the possibility to simulate or not humans with Turing machines (i.e. algorithms, digital computations), including the present intense disputes on the likelihood and conditions of an Artificial Intelligence, has taken place and takes place within this machine-bound frame. Therefore, that whole debate is deeply deformed and contrived. Sometimes grotesque: see the bitter man vs. machine competition to see which does what best and so to guess what jobs machines will steal from humans, or the "art made by AI" neo-modernist hype.

2) Behavior can be judged "intelligent" only by human beings. Therefore, if humans state that the presence of a living body is irrelevant for the purposes of "intelligent" behavior, then by extension they consider a living body irrelevant for the purposes of communication between themselves. Precisely like it is irrelevant between machines. By the way, it is worth noting how this view continues, in a contemporary form, the ancient Platonic-Augustinian creed that there be a "true knowledge" which only can be attained by the intangible intellect/soul, while the material body and its vulgar emotions are just a burden or an atonement. As we just saw, "true knowledge" had definitely took the mathematical turn in the $17^{\text {th }}$ century.

The purported irrelevance of the body is being even reinforced recently by the linguistic realization that "virtual" experiences - those in which the physical body is not present at an event but receives only some informational simulacrum of it - are no less "real" than those experienced in physical presence. Nowadays almost everyone would admit that virtual experiences do lack something, with respect to in-presence experiences; but what is missing, and how important it is for us, are still lesser subjects receiving little attention, at best in narrative ways.

\footnotetext{
2 «Frequently the messages have meaning; that is they refer to or are correlated according to some system with certain physical or conceptual entities. These semantic aspects of communication are irrelevant to the engineering problem» (ivi).

${ }^{3}$ Martin Heidegger started to notice that in his Zur Frage nach der Bestimmung der Sache des Denkens (1968): «Moreover, the cybernetic leading concept of information is far-reaching enough to make one day the historical humanistic disciplines, too, subject to the claim of cybernetics. This comes about all the easier as the relation of the contemporary human being to the historical tradition is increasingly transformed into a mere need for information». Trans. by Richard Capobianco and Marie Göbel.
} 


\section{THE MACHINE-B(I)ASED CULTURE}

Here's what we can read in an outstanding and authoritative study about computational complexity and its philosophical implications (Aaronson 2011).

In practice, people judge each other to be conscious after interacting for a very short time, perhaps as little as a few seconds. This suggests that we can put a finite upper bound - to be generous, let us say $10^{20}$ - on the number of bits of information that two people A and B would ever realistically exchange, before A had amassed enough evidence to conclude B was conscious.

Needless to say, humans don't do this kind of police investigation when they meet ${ }^{4}$. How often in your life have you been wondering whether a new acquaintance was conscious or not? This is a tacit inference that my body is capable of doing by itself, without conscience or reasoning. My body does it immediately when it sees your body, recognizing a body so similar to itself (Rizzolatti 2006). A glance, a gesture, minimal, impalpable and ineffable things, are more than enough to establish meaningful mirroring and communication between A and B. Instead, the computation specialist sees human interaction in terms of Shannon's meaningless bits of information, including those necessary to prove one another that we are conscious beings. Or rather, I should say, to tag another person with the label "conscious".

Notice how this implies a) a definition of "conscious" as a Boolean property (yes/no, 1/0) without nuances, and b) a representation of consciousness as a step function ${ }^{6}$. Once again, formal definitions that sound blatantly unrealistic and out of place referred to humans, not only far from our common sense, but also and above all devoid of scientific grounds. This vision demonstrates how the phylum of formalism keeps advancing in the human domain, pushing us away from (our) nature as it pushes on. The computation specialist shows the way: this is the direction we are drawn by the spread of digital technology and the information society. ${ }^{7}$

In this machine-based environment, the precious natural mirroring capabilities of our bodies are made totally useless. It's a strictly teleological realm of formal language. Everything must be explicitly and exactly descripted and/ or calculated.

The machine-biased culture we are nurturing borrows the same mechanics: it requires us to represent, manage, manipulate, preserve, let's say think, all knowledge as information, that is as digital data, for the sake of computing machinery efficiency. However, we should face the fact that when knowledge is formalized, in any manner, it is as good as data-digitization is just choosing a specific code. In the case of digital data, the choice was motivated by the channel efficiency studied by Shannon.

There's a growing evidence we are getting more and more accustomed to this vision. And this is no surprise for at least two reasons.

A first trivial reason is that humans can adapt to machines far more easily than the other way round. If we are required to pronounce words like robots to be understood by an IVR, we just do it; if we are offered no other cure to loneliness than a robot, we will take that, too; and so on. But not without consequences for our psyche, of course.

The second reason is that the efficiency of information machines is the logical evolution of the previous concept of efficiency, defined by math-wrought economics, which has ruled the world subdued by Western thought for more than a century. Both concepts share a non-human attitude to problem solving, which produces inhuman "solutions" by design.

\footnotetext{
${ }^{4}$ Except for certain known mental disabilities, or a cognitive scientist at work.

${ }^{5}$ This is literally «attaching a name tag to something», in Wittgenstein's words (Ph. Unter.).

${ }^{6}$ Intuitively (and in a simplified definition) a step function's value is 0 below a given value of the free variable, 1 above it.

${ }^{7}$ In a footnote from the same passage, Aaronson says: «People interacting over the Internet, via email or instant messages, regularly judge each other to be humans rather than spam-bots after exchanging a much smaller number of bits!» See how the focus stays on the bodyless textual interactions of the Turing test, describing a human social environment in the image and likeness of (computing) machines.
} 
The normality of digital data to our eyes is such that sometimes even serious opposition to certain downsides of algorithms and AI, for example gender or race biases, may fail to see the effect of the bigger picture they are framed into: a machine-b(i)ased culture/society, which is inherently toxic and oppressive for humans, and is a perfect framework for plenty of novel kinds of oppression.

What is left out of a machine-b(i)ased culture/society? What are we losing in this transformation? I will remark here two key points among others.

From an epistemological point of view, the machine-b(i)ased environment tends to neglect, remove, suppress all non-formalized human knowledge, i.e. tacit/personal knowledge, explicit informal knowledge, knowledge containing ambiguity, narrative, art, etc. For one simple reason: it has no means to represent it.

In social terms, the machine-b(i)ased culture pushes off our natural relational skills, an extremely sophisticated neurobiological apparatus forged by millions of years of evolution. Lately, neuroscience has been shedding much light on how this knowledge generator, rooted into the sensorimotor apparatus (Gallese, Lakoff $2005)$, creates an "embodied simulation" of others within ourselves, and a sort of "intersubjective resonance" that makes the individual an organic part of a group. This is the very fabric of human society. Empathy (Cohen 2011), care, trust, reputation, reciprocity, joint commitment (Tomasello 2016), recognition (Honneth 1992), belonging (Berlin 1958, 1969) - all of these work in the direct relationship between bodies, beneath consciousness and upstream of language.

By erasing the founding forms of knowledge and communication, the machine-b(i)ased culture brings a radical impoverishment endangering our sense-making and the proper operation of human societies.

\section{DATA AS AN «AUTOMATIC FETISH»}

I believe that Marx's piercing analysis of money as a dehumanizing abstraction and automatically replicating capital can be extended with no major changes to the main abstraction and «automatic fetish» of our historical regime: digital data. This is why rewriting Marx makes sense.

Let's test this idea further by rewriting two passages of Marx's Capital. In this first excerpt he compares two dynamics of relationship between people, money and commodities.

The simplest form of the circulation of commodities is C-M-C, the transformation of commodities into money, and the change of the money back again into commodities; or selling in order to buy. But alongside of this form we find another specifically different form: M-C-M, the transformation of money into commodities, and the change of commodities back again into money; or buying in order to sell. Money that circulates in the latter manner is thereby transformed into, becomes capital, and is already potentially capital. [...] The circuit C-M-C starts with one commodity, and finishes with another, which falls out of circulation and into consumption. Consumption, the satisfaction of wants, in one word, use-value, is its end and aim. The circuit M-C-M, on the contrary, commences with money and ends with money. Its leading motive, and the goal that attracts it, is therefore mere exchange-value (Marx 1887).

Again, I will replace the commodity $C$ with the knowledge akin to the personal enjoyment of goods, that is the "personal knowledge": subjective experience that is embodied as some knowledge. Concrete, limited, situated, ineffable, etc. I will denote it by $H$, for "Human", in order to emphasize the contrast with machines (non-H).

The "use-value" is common to both $C$ and $H$ : the use is always a personal experience, when one utilizes a commodity as well as when one consumes digital content, and that experience can generate meaningful personal knowledge.

"Consumption" has its equivalent in the "existence", for personal knowledge is deeply part of how an individual lives his/her life.

Recalling what I said above, we have various ways to exchange personal knowledge. As in Marx the primordial economic exchange is C-C, so the tacit/personal knowledge can be transmitted between us in the form $\mathrm{H}-\mathrm{H}$, in physical presence, by direct and unconscious imitation between bodies, as between parents and children or 
between teachers and students. ${ }^{8}$ Then we have the explicit, discursive, narrative, analogical knowledge, represented through some living and informal language: for example, cultural norms, literature, scientific theories, etc. If we denote it with $K$, we have the circulation $\mathrm{H}-\mathrm{K}-\mathrm{H}$ in which knowledge handover is mediated by linguistic artifacts. Eventually, in the modern age $K$ shifts inexorably towards $D$, that is formalized knowledge, convertible or converted into digital data. The circulation becomes H-D-H where $\mathrm{D}$ assumes the same role of money in Marx, what «mediates the existence of other people for me».?

What is the equivalent of "exchange-value" in the new domain? Comparing the way data works to the way money works, the exchange-value represented by data is actually its abstract functionality, i.e. the capacity to accomplish a given function, and impersonal fungibility, i.e. the property of being interchangeable. Not by chance both these properties are parts of the traditional efficiency concept mentioned above. ${ }^{10}$

Hence, we can rewrite as follows:

\begin{abstract}
* The simplest form of the circulation of knowledge is $\mathrm{H}-\mathrm{D}-\mathrm{H}$, the transformation of personal knowledge into data, and the change of data back again into personal knowledge. But alongside of this form we find another specifically different form: D-H-D, the transformation of data into personal knowledge, and the change of personal knowledge back again into data. Data that circulates in the latter manner is thereby transformed into, becomes capital, and is already potentially capital. [...] The circuit H-D-H starts with one's personal knowledge, and finishes with another one's, which falls out of circulation and into existence. Existence, the satisfaction of wants, in one word, use-value, is its end and aim. The circuit D-H-D, on the contrary, commences with data and ends with data. Its leading motives, and the goals that attract it, are therefore mere functionality and fungibility.
\end{abstract}

As you may have noticed, "capital" remained as a fixed point of the transformation. This will deserve an indepth analysis, because money and data are not really separate scopes but interwoven processes, melding Marx's original universe with my transposition defined by the replaced concepts. In fact, data can be many things, among which:

a) a commodity that can be bought and sold;

b) a representation of money;

c) a product of labor;

d) self-replicating value.

This twist is extremely interesting and opens up a whole world, too vast to explore it here. For the moment being, I will just treat the subject (d) and therefore proceed to the crucial passage in which Marx defines money as capital. Here is the original quote, coming right after the last one.

In the simple circulation of commodities [C-M-C], the two extremes of the circuit have the same economic form. They are both commodities, and commodities of equal value. But they are also use-values differing in their qualities, as, for example, corn and clothes. The exchange of products, of the different materials in which the labour of society is embodied, forms here the basis of the movement. It is otherwise in the circulation M-C-M, which at first sight appears purposeless, because tautological. Both extremes have the same economic form. They are both money, and therefore are not qualitatively different use-values; for money is but the converted form of commodities, in which their particular use-values vanish. [...] One sum of money is distinguishable from another only by its amount. The character and tendency of the process M-C-M, is therefore not due to any qualitative difference between its extremes, both being money, but solely to their quantitative difference. More money is withdrawn from circulation at the finish than was thrown into it at the start. The cotton that was bought for $£ 100$ is perhaps resold for $£ 100+£ 10$ or $£ 110$. The exact form of this process is therefore M-C-M', where $\mathrm{M}^{\prime}=\mathrm{M}+\Delta \mathrm{M}=$ the original sum advanced, plus an increment. This increment or excess over the original value I call "surplus-value". The value originally advanced, therefore, not only remains intact while in circulation, but adds to itself a surplus-value or expands itself. It is this movement that converts it into capital.

Let's try and rewrite this straight, saving the necessary clarification for later.

\footnotetext{
${ }^{8}$ In fact, «it can be proven that in human beings all types of learning - conditioning, learning of motor skills and instrumental learning or operant conditioning - take place without any awareness or contribution from consciousness» (Jaynes 2014).

${ }^{9}$ See note 1.

${ }^{10}$ Compare with Heidegger's "Zuhandenheit" or "usability"; see also note 3.
} 
* In the simple circulation of knowledge [H-D-H], the two extremes of the circuit have the same existential form. They are both personal knowledge, of akin value. But they are also use-values differing in their qualities, as, for example, drawing a flower and cooking an egg. The exchange of knowledge, of the different kinds that embody in the labour of society, forms here the basis of the movement. It is otherwise in the circulation D-H-D, which at first sight appears purposeless, because tautological. Both extremes have the same form. They are both data, and therefore are not qualitatively different use-values; for data is but the converted form of personal knowledge, in which its particular use-values vanish. [...] One load of data is distinguishable from another only by its amount. The character and tendency of the process D-H-D, is therefore not due to any qualitative difference between its extremes, both being data, but solely to their quantitative difference. More data is withdrawn from circulation at the finish than was thrown into it at the start. The digital content that was $1 \mathrm{MB}$ in size is perhaps returned as $1 \mathrm{MB}+1 \mathrm{~KB}$. The exact form of this process is therefore $\mathrm{D}-\mathrm{H}-\mathrm{D}$ ', where $\mathrm{D}^{\prime}=\mathrm{D}+\Delta \mathrm{D}=$ the original data, plus an increment. This increment or excess over the original value I call " $\mathrm{X}$ ”. The value originally advanced, therefore, not only remains intact while in circulation, but adds to itself a X or expands itself. It is this movement that converts it into capital.

I left the replacement for "surplus value" open with an X; we will get there in a moment.

In the information society we experience infinite occurrences of the circulation H-D-H, for example when we chat with someone through a social network (different starting and ending H's), or when we retrieve content with a search engine (same starting and ending $\mathrm{H}$ ).

To understand the circulation D-H-D correctly, we must bear in mind that in a machine-based environment everything is being beheld from a machine point of view. Data, from a machine point of view, is just bitsets. It's not "meaningful" in any sense we give to this word. Paradoxically, meaning is meaningless to machines. Only humans can extract "meaning" from data. They do it compulsively and they want it desperately, for it's the very heart of their existence. So, we must now resist the strong temptation to interpret the D endpoint in the circulation D-H$\mathrm{D}$ as a potential signifier, like some content a person can enjoy. If this were the case, the actual cycle here would be (D-)H-D-H, that is the first one, "the simple circulation of knowledge". It's exactly like in Marx's M-C-M process: the second $\mathrm{M}$ is not money allocated to buy some commodity, otherwise we would fall back to the "healthy" circulation C-M-C.

In Marx the ending $\mathrm{M}$ in $\mathrm{M}-\mathrm{C}-\mathrm{M}$ is sheer money, a number; in our case the ending $\mathrm{D}$ in $\mathrm{D}-\mathrm{H}-\mathrm{D}$ is sheer data, a bitset-again, a number. And one bitset is «distinguishable from another only by its amount», that is another number, a value computed from that data: its length in bytes, a checksum, a hash function, etc.

What's that odd $1 \mathrm{~KB}$ added to the original $1 \mathrm{MB}$ of consumed content? It's metadata. In the circulation D-H$\mathrm{D}$ ', the human middle $\mathrm{H}$ between $\mathrm{D}$ and $\mathrm{D}$ ' is used to beget that $\Delta \mathrm{D}$ of metadata that expands the value of original data by adding something human, that is something meaningful to us. Once gone over to the D domain, that something ultimately serves to increase the above said efficiency and hence the economic value of the data. Its relationship with humanity is left as a very vague residue (a «merely imperfect form», see "price" on page 203).

Metadata can be generated by humans indirectly through their interactions with machines: top search keywords and trend topics, web navigation patterns, reactions on social networks, geolocation of photos and videos, etc. Or else they can be generated directly, manually, i.e. by inputting values. ${ }^{11}$ Among the latters, annotations or labels that associate meanings to the training datasets needed by AI development are gaining a fundamental role.

Such metadata are highly significant for two reasons: a) they shape the interiors of deep networks black boxes, and b) more and more they are produced by unskilled and exploited gig workers of a newborn, specific industry. This industry is growing fast in developing countries because it's vital to keep AI's promises. ${ }^{12}$ We have here a telling anticipation of how the sinister D-H-D' loop closes on itself in a triple technical, social, and economic way.

Handmade data annotation/labelling is as machine-biased as the Turing test. Albeit performed by humans, it is indeed a computer function: it permanently associates to some human-readable content a defined set of tags, that

\footnotetext{
${ }^{11}$ However, this distinction does not exist from a machine perspective. If a label, which is text, is considered metadata of a piece of content when it is associated with it, then a comment on a Facebook post (for example) can also be considered metadata of that post, while for us it is part of a conversation. In concrete terms, what is or is not metadata depends on the data structure defined in the code.

${ }^{12}$ https://medium.com/financial-times/ais-new-workforce-the-data-labelling-industry-spreads-globally-f472cb1bac09
} 
is a formal restriction of natural language. ${ }^{13}$ After this operation, the digital content becomes entirely autonomous, in the sense that it assumes a closed form, allegedly freed from the need of any further human intervention. Now that data is usable, fungible, formalized knowledge. But it incorporates once and for all one particular interpretation (meaning), that one given by who annotated it. From now on it, wherever that data + metadata come from, it will be accessible only through that interpretation. Whoever uses it will be locked in that interpretation.

Since interpretative metadata are always the result of someone's cognitive work, they do carry some subjectivity with them. But in passing through the D-H-D' pipeline the shadow of this subjectivity apparently evaporates forever in the digital light, once the interpreted content is absorbed in D' and thus crystallized, made "objective". In Marx's words, «the intermediate steps of the process vanish in the result and leave no trace behind».14

Consider, as an example, the ImageNet database. ${ }^{15}$ It's a corpus of datified knowledge made of more than 14 million labelled images that is used as-is by researchers from all over the world to train and test neural networks for visual object recognition. Like with many other benchmark datasets of the same kind, its knowledge is employed without any reference to where and whom labels come from: the middle humans in the D-H-D' labeling have disappeared. The process has apparently become D-D' as if the labels were native properties of the raw data.

Yet those labels are not negligible accessories. They have a primary role in calculating the loss function used to update weights in the deep networks, thus driving the learning and improvement process up to an AI model deployment. They profoundly affect the future AI model behavior. Thus, the vanishing of their origin means a naturalization and legitimation of potentially harmful choices.

Researchers who employ ImageNet to train deep neural networks are the H's who constitute a new D-H-D' cycle, as they figure out, test, and execute the right training algorithms. Once trained, the network is set to work. From that moment on it cranks out its own judgments autonomously, because this is precisely the purpose it has been made for. Once again, $\mathrm{H}$ has disappeared and the process is reduced to D-D': data generating more data.

For Marx, the ultimate evolution of money-capital is the self-reproduction M-M': money growing indefinitely by itself by an interest rate, without the need to turn into human-bound commodities any longer, and thus bringing a non-human devastating principle at the heart of human society. Similarly, data tends to its direct self-reproduction D-D' without the need to mean something to us, or (which is the same) being really useful to us any longer.

Data is acquiring the ultimate ability to grow autonomously and uncontrollably, thanks to big data and machine learning methodologies. AI is the technology enabling D-D' to the highest degree, especially the «artificial intuition» that Deep Learning is more and more capable of (Perez 2018). Unsupervised and self-play types of learning are the extreme manifestations of the D-D' trend, achieving the uncanniest feats. ${ }^{16}$

"Autonomous" is the keyword of D-D'. We may say that "autonomous" means D-D'. We may even say that "autonomous" has always meant D-D' well before AI and digital computation came to light. By getting rid of the last "human in the loop", the D-D' cycle fulfills the ancient ideal that always guided the big phylum of formalism introduced above: which is to expel the human factor from the formation of knowledge and from decisional processes altogether. Why? Because some people ${ }^{17}$ have always seen typical humans as flawed: not logical enough, prone to infinite mistakes, spoiled by their emotions, impaired by the corruption and death of their bodies. Therefore, according to those people, humans must be corrected, or rather, replaced, by the perfection of mathematics and of machines. This ideology is still alive and kicking, well apparent in arguments such as those supporting selfdriving cars or transhumanism. ${ }^{18}$

\footnotetext{
${ }^{13}$ Once again, «attaching a name tag to something» (see note 5$)$.

${ }^{14}$ K. Marx, Capital, ibid.

${ }^{15}$ Created by Fei Fei Li et al.

${ }^{16}$ See the GPT-3 language model by OpenAI, AlphaZero by DeepMind, Pluribus by Facebook \& Carnegie Mellon University, Generative Adversarial Network (GAN) architectures.

${ }^{17}$ Arguably they are to be found among hypersystemizers with scant empathy, see S. Baron-Cohen (2020).,

18 «When we have succeeded in automating computer science and artificial intelligence research, » says Nate [Soares], «the feedback loop will close, and systems will appear that can build better systems on their own» (O'Connell 2017).
} 
The omnipotence of money $\mathrm{M}$ that can buy everything, told by Marx, change into the omnipotence of data $\mathrm{D}$ that can represent everything. ${ }^{19}$ Data power is no longer a material appropriation over things and people, but rather something more subtle and abstract, more general and devious. It's not the universality of property, it's the universality of knowledge, and above all knowledge about human beings.

It is often said, "machines know us better than we know ourselves". Although this is a myth, an optical illusion that can be easily demolished, the story holds up. It persuades and intimidates. Eventually, when the most dangerous D-D' application, that is profiling, creates a $\Delta \mathrm{D}$ about me that is just a computed fabrication but nevertheless has real effects, like taking away credit, work, liberty, then the ghastly power of data becomes tangible. But in the meantime, it goes on acting covertly in a myriad of interconnected software systems.

\section{«TWO ROADS DIVERGED IN A WOOD»}

At the very end of an AI-powered D-D' cycle, the AI output D' may follow two roads.

A. Take a descriptive role: feed a decision support system in a mixed man-machine environment designed to help humans in a sensible way. ${ }^{20}$ In other words, close the circulation with $-\mathrm{H}$.

B. Take a performative role: make autonomous decisions that have actual bearings on people's lives, in fact acting like a proxy law system and an independent, inscrutable, often private executive power. Here the circulation closes with -D.

$A$ is the "healthy" cycle, the safest and most reasonable path. At least because it takes well into account a basic anthropological truth: our deepest interests as humans (and, in a broader scope, as parts of nature) should be the endpoint of every artificial process we build.

$B$ is a slippery slope leading to the obscure apotheosis of the machine-b(i)ased culture. As the augmented D' expands, what do its meaning and authentic value become? Will D' really have something to do with us, with our interests as humans? We have no idea. D' is purely empirical, the AI procedures that generate D' are unverifiable, oracular, transcendent. They must be simply accepted as they come. They require a blind faith in the machine. This necessary faith makes the information society a deeply transcendental society.

All the infinite $\Delta \mathrm{D}$ increases add together and combine, giving a violent acceleration plus an abstraction boost to the human society. The double "expansion" of acceleration and abstraction can be seen as the equivalent, in the data regime, of Marx's "surplus value" - that X I had left hanging in the last rewrite. Their combined effect is to vertiginously detach us from reality ${ }^{21}$, from the meaning of life, and from one another as humans. When we see one another through machines, we see one another as machines see us-that is, as things.

In conclusion, here's a summary of my theses in this work:

- Digital data can be seen as a generalization of Marxian money, replacing it as the ultimate, universal abstraction and intermediary between humans.

- This change meets the need, for capitalism, to escalate to a higher level of abstraction, in order to shift to a form that offer less resistance in the present conditions.

- The data economy is a generalization of the capitalist economy, where money itself has become data. A datadriven society is a seamless continuation of a market-driven society.

\footnotetext{
${ }^{19}$ «The information society is more accurately understood as a neo-manufacturing society in which energy and raw materials have been supplanted by data and information as the new digital gold and the true source of added value»(Floridi 2014).

${ }^{20}$ See for example Cabitza, Campagner ., Ciucci ., Seveso (2019)

${ }^{21}$ Emblematic, in this sense, are AWS (autonomous weapon systems) and HFT (high-frequency trading): they are computational versions of two ancient human activities from which humans themselves are now excluded precisely because of the inhuman speed of operations, with consequences that are hard to imagine. Equally emblematic is the difficulty in attributing materiality and therefore an ecological footprint to everything that happens "in the cloud".
} 
- The information society (the culture based on data and the machines that process it) features an idolatry of data that can be seen as an updated, extreme form of the «idolatry of fact».22

- Just as capitalism was not born to pursue the goal of self-replicating money, so the information society was not born to pursue the goal of self-replicating data. Self-replication is a consequence of a certain socio-technical design, inspired by a specific abstract, simplified, quite unreal vision of man.

- Money-capital is a historically determined means to exercise oppressive power. Data-capital is another. Being unlikely that machines will ever "behave humanely with us" (see epigraph) now or in any imaginable future, data-capital promises to be worse. If there is one thing machines are perfect for, it's oppressing humans.

- Not opposing this drift, or depicting the future as if it were already data-written, is a novel laissez-faire that is bound to even greater disasters than the laissez-faire we already endured.

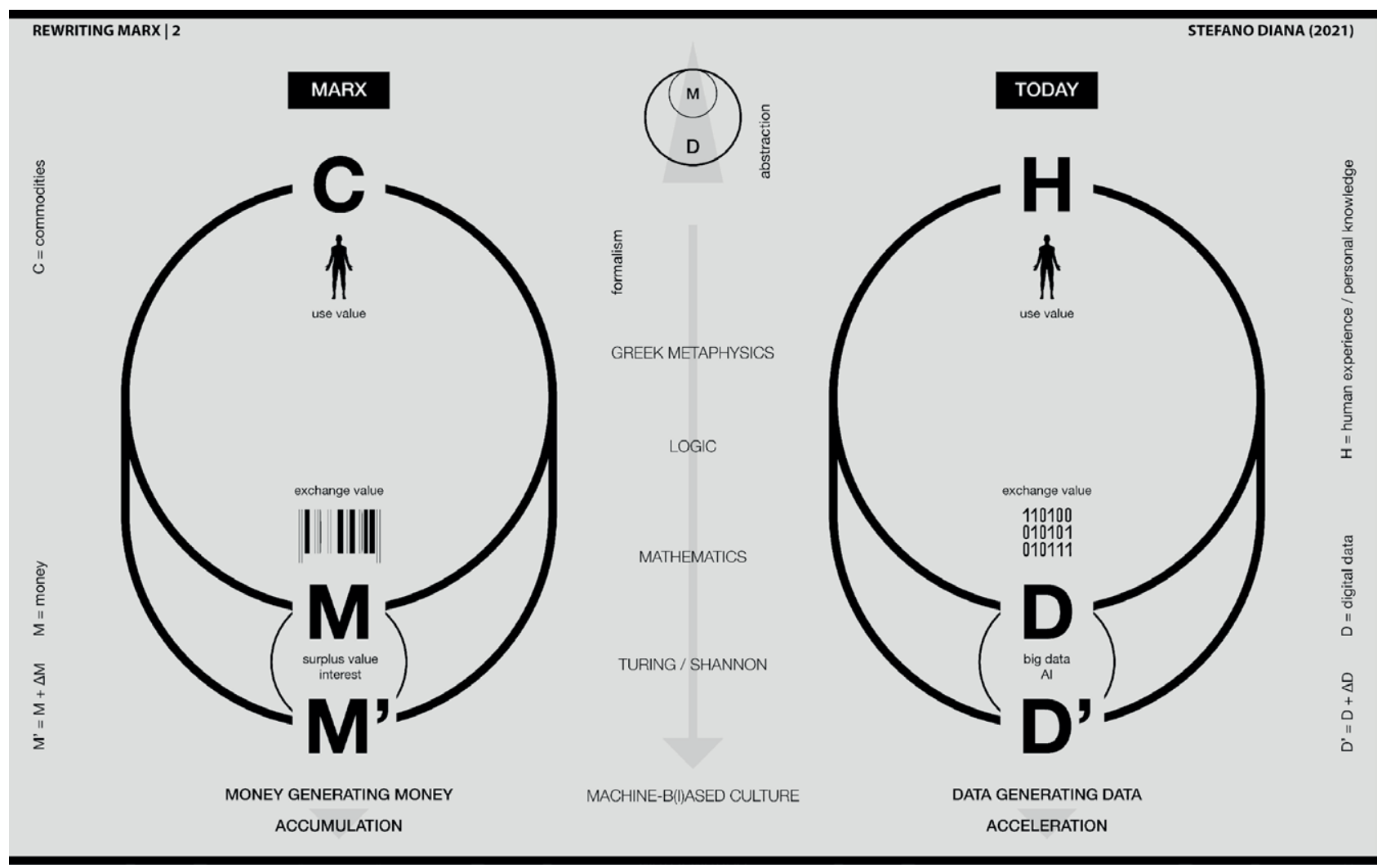

\section{BIBLIOGRAFIA}

Aaronson, S. (2011). Why philosophers should care about computational complexity.

Baron, S. B. (2020). The Pattern Seekers: A New Theory of Human Invention,

Penguin Books.

Baron-Cohen, S. (2011). Zero degrees of empathy: A new theory of human cruelty. Penguin uk.

Barthes, R. (1957). Lemythe aujourd'hui, in «Mithologies».

Berlin, I. (1958, 1969). Two Concepts of Liberty.

${ }^{22}$ Denounced by Nietzsche as an active substance of historicism, which indeed belongs to the phylum of formalism. See F. Nietzsche (1874). 
Cabitza, F., Campagner, A., Ciucci, D., \& Seveso, A. (2019, September). Programmed inefficiencies in DSS-supported human decision making. In International Conference on Modeling Decisions for Artificial Intelligence (pp. 201-212). Springer, Cham.

Changeux, J.P. (2004). L'homme de verité.

Gallese, V., \& Lakoff, G. (2005). The brain's concepts: The role of the sensory-motor system in conceptual knowledge. Cognitive neuropsychology, 22(3-4), 455-479.

Heidegger, M. (1968). Zur Frage nach der Bestimmung der Sache des Denkens.

Honneth, A. (1992). Kampf um anerkennung. Zur moralischen grammatik sozialer konflikte. Frankfurt am Main: Suhrkamp.

Israel,G.(1966). La Visione Matematica della Realtà.

Jaynes, J. (2014). The Diachronic Nature of Consciousness.

Marx, K. (1844). Comments on James Mill, Éléments d'économie politique: Collected works.

Marx, K. (1887). A Critique of Political Economy.

Marx, K. (1973). Grundrisse: Foundations of the critique of political economy. Penguin UK in association with New Left Review.

Marxm K. (1844). Economic and Philosophic Manuscripts.

Nietzsche, F. (1874). Unzeitgemässe Betrachtungen. Zweites Stück: Vom Nutzen und Nachtheil der Historie für das Leben.

O’Connell, M. (2017). Being a Machine.

Perez, C. E. (2018). Artificial Intuition: The Improbable Deep Learning Revolution. Carlos E. Perez.

Polanyi, M. (1958). Personal knowledge: Towards a Post-Critical Philosophy.

Polanyi, M. (1966). The Tacit Dimension.

Rizzolatti, G., \& Sinigaglia, C. (2006). So quel che fai: il cervello che agisce ei neuroni specchio. Milano: R. Cortina.

Shannon, C. E. (1948). A mathematical theory of communication. The Bell system technical journal, 27(3), 379423.

Tomasello, M. (2016). A natural history of human morality. Harvard University Press.

Turing, A. M. (1936-1937). On computable numbers, with an application to the Entscheidungsproblem. J. of Math, 58(345-363), 5.

Turing, A. M. (1950). Computing machinery and intelligence. In Mind (pp. 433-460).

Wittgenstein, L. (1953). Philosophische Untersuchungen. 